Submitted to ApJ

\title{
Resonant inclination excitation of migrating giant planets
}

\author{
Edward W. Thommes \\ Astronomy Department, University of California, Berkeley, CA 94720 \\ and \\ Jack J. Lissauer \\ Space Science Division, MS 245-3, NASA-Ames Research Center, Moffett Field, CA 94035
}

\begin{abstract}
The observed orbits of extrasolar planets suggest that many giant planets migrate a considerable distance towards their parent star as a result of interactions with the protoplanetary disk, and that some of these planets become trapped in eccentricity-exciting mean motion resonances with one another during this migration. Using three-dimensional numerical simulations, we find that as long as the timescale for damping of the planets' eccentricities by the disk is close to or longer than the disk-induced migration timescale, and the outer planet is more than half the mass of the inner, resonant inclination excitation will also occur. Neither the addition of a (simple, fixed) disk potential, nor the introduction of a massive inner planetary system, inhibit entry into the inclination resonance. Therefore, such a mechanism may not be uncommon in the early evolution of a planetary system, and a significant fraction of exoplanetary systems may turn out to be non-coplanar.
\end{abstract}

\section{Introduction}

The orbital migration of bodies locked in mean-motion resonances is a major recurring theme in the early history of the Solar System. A review of resonance dynamics in the Solar System is given by Peale (1976). Goldreich (1965) showed that tidally-induced orbital migration is likely responsible for most of the mean-motion commensurabilities among the satellites of Jupiter and Saturn. In the Uranian satellite system, tidally-induced convergent migration of the moons Miranda and Umbriel could have led to capture into, and subsequent 
escape from, inclination-type resonances, thus producing Miranda's anomalously high orbital inclination of $4.3^{\circ}$ (Tittemore \& Wisdom 1989; Malhotra \& Dermott 1990).

In the case of the outer Solar System, Malhotra (1998) and Gomes (2000) showed that bodies entrained in the 2:3 exterior mean-motion resonance of an outward-migrating Neptune can also encounter the Kozai resonance, as well as secular and mean-motion inclination resonances. These resonances increase inclination, and this may be the mechanism by which Pluto and the Kuiper belt objects presently sharing the 2:3 resonance (plutinos) attained their high inclinations.

Snellgrove et al. (2001) and Lee \& Peale (2002) performed in-depth investigations of resonant capture and migration in the context of the GJ 876 planetary system. They found that convergent orbital migration of planets typically leads to their capture into the 2:1 meanmotion resonance. The resonant lock thus produced is characterized by the libration of the first-order resonance angles (cosine arguments in the expansion of the disturbing function; see for example Murray \& Dermott (1999)),

$$
\begin{aligned}
& \theta_{e_{1}} \equiv \lambda_{1}-2 \lambda_{2}+\tilde{\omega}_{1} \quad \text { and } \\
& \theta_{e_{2}} \equiv \lambda_{1}-2 \lambda_{2}+\tilde{\omega}_{2}
\end{aligned}
$$

about zero degrees, where $\lambda_{i}$ is the $i$ th planet's longitude, $\tilde{\omega}_{i}$ is its longitude of pericenter, and $i=1,2$ refer to the inner and outer planet, respectively. Since $\theta_{e_{1}}-\theta_{e_{2}}=\tilde{\omega}_{1}-\tilde{\omega}_{2}$, the zeroth-order secular resonance angle

$$
\theta_{\tilde{\omega}} \equiv \tilde{\omega}_{1}-\tilde{\omega}_{2}
$$

also librates about zero; in other words, the orbits of the two planets librate about apsidal alignment.

The analysis of Lee \& Peale (2002) is restricted to the planar case, thus the effects of migration in resonance on orbital inclinations are not considered. Starting with slightly non-coplanar orbits, we initially see the same behaviour as reported by Lee \& Peale (2002): In the absence of any damping mechanism, the eccentricities of both bodies grow. However, we find that once the inner planet's eccentricity is high enough, the system will also enter an inclination-type resonance, which induces rapid growth in the inclinations of both planets.

In this article, we report the results of three-dimensional simulations of migrating planets in resonance. We give a summary of the underlying physical picture in Section 2. The methods that we use, as well as initial conditions, are presented in Section 3. Baseline cases with two equal mass planets are discussed in Section 4. We extend our analysis to planets of unequal masses in Section 6, impose eccentricity damping in Section 7, include 
the gravitational potential of the disk in Section 8, and add four Uranus/Neptune mass inner planets in Section 9. We conclude in Section 10 with a summary of our results and a discussion of their implications.

\section{The physical picture}

Gas giant planets must form in a protoplanetary disk which has not yet lost its gas; this constrains the time of formation to within, approximately, the first $10^{7}$ years of the disk's lifetime (Strom, Edwards, \& Skrutskie 1990; Haisch, Lada, \& Lada 2001). If gas giants form by the accretion of a massive atmosphere onto a $\sim 10 \mathrm{M}_{\oplus}$ solid core (Mizuno, Nakazawa, \& Hayashi 1978; Pollack et al. 1996; Bodenheimer et al. 2000), then the most favourable location for such large cores to form by planetesimal accretion is in an annulus several AU wide beyond the ice-condensation line, roughly the location of Jupiter and Saturn in our own Solar System (Thommes, Duncan, \& Levison 2003). A gas giant-sized body (mass of order $10^{2} \mathrm{M}_{\oplus}$ ) will open a gap in the gas disk. Its orbital evolution will subsequently be locked in to the viscous evolution of the gas disk, as long as the disk mass is large compared to that of the planet. This is commonly referred to as Type II migration (Ward 1997); see Lin et al. (2000) or Thommes \& Lissauer (2003) for a review. In this picture, significant Type II migration probably did not occur in our Solar System; however, this mechanism is likely to have played a significant role for those extrasolar giant planets with orbital semimajor axes of less than a few AU. For a review of discovered extrasolar planets and the statistics of their orbits, see Cumming et al. (2003).

If a disk forms two gas giants, the annulus of gas between their individual gaps may be relatively rapidly depleted, so that both planets end up sharing a common gap (Kley 2000; Bryden et al. 2000) Also, if the viscosity is higher and thus the accretion of the disk onto the star faster at smaller radii, as in the model of Gammie (1996), the inner disk may be depleted on a timescale short compared to the migration timescale of the planets.

\section{Numerical methods}

We use a variant of the SyMBA integrator (Duncan, Levison, \& Lee 1998), which has the desirable properties of the mixed-variable symplectic (MVS) mapping of Wisdom \& Holman (1991), and in addition handles close encounters among objects by employing a variable timestep technique. We impose an inward migration on the outer planet, in a way which simulates interaction with a viscously evolving outer gas disk: We define an inner radius 
$r_{\text {disk }}$ to the gas disk; this radius is made to change at a rate $\dot{r}_{\text {disk }}$. The semimajor axis of the outer planet, $a_{2}$, is then made to change based on its location relative to the gap edge:

$$
\dot{a}_{2}= \begin{cases}-S\left(a_{2}-r_{\text {disk }}\right), & a_{2}>r_{\text {disk }} \\ 0, & a_{2}<r_{\text {disk }}\end{cases}
$$

where $S$ is large and positive, so that the magnitude of $\dot{a}_{2}$ increases steeply outside of $r_{\text {disk }}$. In this way, a planet with $a_{2}>r_{\text {disk }}$ is always constrained to move inward at a mean rate $\dot{r}_{\text {disk }}$; if the torque required to maintain this migration rate changes (as happens when the planet resonantly captures an interior body which it then has to push along), the planet's position relative to the gap edge simply re-adjusts until its orbital decay rate again matches $\dot{r}_{\text {disk }}$. It should be noted that, strictly speaking, $r_{\text {disk }}$ corresponds not to the edge of the disk itself but rather to the location outwards of which a planet is subject to a nonzero disk torque.

The speed with which the disk edge moves inward corresponds to the radial accretion speed of the disk. This is given by

$$
\dot{r}_{\text {accr }} \sim C_{1} \alpha(r \Omega)(c / r \Omega)^{2},
$$

where $\Omega$ is the Keplerian angular frequency, $c$ is the gas sound speed, $C_{1}$ is a constant of order unity, and $\alpha$ is the Shakura-Sunyaev viscosity parameter: $\nu \sim \alpha c^{2} / \Omega$ (e.g., Ward 1997). The factor $(c / r \Omega)$ is also the aspect ratio $h$ of the disk, where $h \equiv H / r ; H$ is the disk scale height at radius $r$. For simplicity we adopt the (flared) aspect ratio of the Hayashi (1981) nebula model, which has $h \propto r^{1 / 4}$; Chiang \& Goldreich (1997) obtain a similar $r$-dependence, $h \propto r^{2 / 7}$, for $r \lesssim 80$ AU. We thus have a disk accretion speed $\dot{r}_{\text {accr }}$ which does not vary with $r$, assuming $\alpha$ is constant. Using $h=0.05(r / \mathrm{AU})^{1 / 4}$, the accretion speed is $\dot{r}_{\text {accr }} \sim 10^{-2} \alpha$ AU/year. The $\alpha$ viscosity of a protoplanetary disk is estimated to be $10^{-4}$ to $10^{-2}$ (e.g., Cabot et al. 1987; Dubrulle 1993). Adopting $\alpha=10^{-3}$, we have $\dot{r}_{\text {accr }} \sim 10^{-5} \mathrm{AU} /$ year.

It should be emphasized that migration is imposed on the outer planet as per Eq. 3 regardless of the eccentricity of the planet, or its inclination relative to the plane of the disk (which is the planet's original orbital plane). Hydrodynamic simulations of the interaction of a significantly eccentric and/or inclined gap-opening planet with a disk have, to our knowledge, not yet been performed, so we operate under the simple assumption that, no matter how the planet's orbit evolves, its semimajor axis remains locked relative to the inner edge of the disk. However, at sufficiently high eccentricity and/or inclination, a planet will very likely no longer be an effective barrier to the disk, so that this assumption would no longer be valid. We will discuss implications in Section 10.

The shortest base timestep used in the simulations is $10^{-3}$ years, and integration is stopped when a body comes within $0.1 \mathrm{AU}$ of the star. Beyond this point, the timestep is 
deemed too long, and thus the azimuthal motion of the inner planet during one timestep too large, to accurately resolve the interactions between the two planets.

In all of the simulations below, we start with a system of two planets on nearly circular orbits about a one solar mass $\left(\mathrm{M}_{\odot}\right)$ star, the inner at $5 \mathrm{AU}$, and the outer at $8.5 \mathrm{AU}$. Initial eccentricities and inclinations are of order $10^{-3}$ and $\left(10^{-2}\right)^{\circ}$, respectively.

\section{The baseline case}

We begin with a system consisting of two Jupiter-mass planets. Several runs are performed, with differing initial orbital phases, but the outcomes are very similar. Fig. 1 shows one example. The outer planet migrates inward until it and the inner planet are mutually captured into the 2:1 mean-motion resonance, with both first-order eccentricity-type resonance angles, $\theta_{e_{1}}$ and $\theta_{e_{2}}$ (Eq. 1), librating about zero, which means that conjunctions are occurring when both planets are at pericenter, and thus that the orbits are also librating about apsidal alignment. This behaviour is to be expected even for planets that start with a larger separation in orbital periods; Lee \& Peale (2002) find that as long as $\dot{a_{2}} \lesssim 10^{-3}(a / \mathrm{AU})^{-1 / 2}$, capture into the $2: 1$ resonance occurs with certainty if the eccentricities of both planets are low $\left(\lesssim 10^{-1}\right)$, whereas larger eccentricities are required for a nonzero capture probability into more distant, higher-order resonances which would be encountered first (such as 3:1).

The eccentricities of both planets increase as they migrate in resonance, which can be understood in terms of energy $(E)$ and angular momentum $(L)$ exchange between the disk and the system of resonantly-locked planets (Lissauer, Peale, \& Cuzzi 1984): In order to change the semimajor axis of a circular orbit while keeping it circular, $E$ and $L$ have to be changed in the right ratio: $\dot{E} / \dot{L}=d E / d L=\sqrt{G M_{*} / a^{3}}$, which is just the orbital angular velocity, $n(a)$. But $n(a)$ is a decreasing function of semimajor axis. Thus, even though we assume a disk torque that changes the orbit of the initially circular outer planet in such a way that $\dot{E} / \dot{L}=n\left(a_{2}\right)$, this ratio is too low for the inner planet. Since $\dot{E}$ and $\dot{L}$ are negative, the two-planet system as a whole acquires an angular momentum deficit and must respond by increasing the eccentricity of one or both of its components. The rate of eccentricity growth can be reduced by making the applied $\dot{E} / \dot{L}$ higher than what would be required to keep the outer planet by itself circular. This would amount to applying eccentricity damping to the outer planet. The evolution of the system with eccentricity damping by the disk is investigated in Section 7.

In Fig. 1E and F, we also plot the resonance angles for the 4:2 inclination-type mean 
motion resonance. This is a second-order resonance (which is the lowest possible order for an inclination resonance); the associated terms in the expansion of the disturbing function are of order $i^{2}$. These angles are defines as

$$
\theta_{i_{1}^{2}}=2 \lambda_{1}-4 \lambda_{2}+2 \Omega_{1}
$$

and

$$
\theta_{i_{2}^{2}}=2 \lambda_{1}-4 \lambda_{2}+2 \Omega_{2}
$$

with $\Omega_{1}, \Omega_{2}$ being the longitudes of the ascending nodes of the inner and outer planet, measured relative to an arbitrary but fixed direction in the inertial frame, and in the plane of the (notional) protoplanetary disk, which is, within an inclination of order $\left(10^{-2}\right)^{\circ}$, also the initial plane of both planets' orbits.

Both angles are initially circulating, but $\dot{\theta_{i_{1}^{2}}} / 2$ and $\dot{\theta_{i_{2}^{2}}} / 2$ decrease over time until, at about $4 \times 10^{5}$ years, $\theta_{i_{1}^{2}} / 2$ starts librating about about $90^{\circ}$, while simultaneously $\theta_{i_{2}^{2}} / 2$ starts librating about $270^{\circ}$. This means that $\theta_{i_{1}^{2}}$ and $\theta_{i_{1}^{2}}$ are both librating about $180^{\circ}$. Fig. $1 \mathrm{D}$ shows that initially, the inclinations of both bodies remain at $\lesssim 0.01^{\circ}$, oscillating but undergoing no net growth. However, as the system enters the inclination resonances, the inclinations of both bodies begin to grow rapidly to $\sim 10^{\circ}$ (the outer planet) and $\sim 20^{\circ}$ (the inner one) in just tens of thousands of years. Thereafter, the inclinations continue to grow more slowly.

This simultaneous libration of $\theta_{i_{1}^{2}}$ and $\theta_{i_{2}^{2}}$ also implies the libration of the "mixed" resonance angle,

$$
\theta_{i_{1} i_{2}}=2 \lambda_{1}-4 \lambda_{2}+\Omega_{1}+\Omega_{2}=\theta_{i_{1}^{2}} / 2+\theta_{i_{2}^{2}} / 2
$$

about $0^{\circ}$, as well as libration of the zeroth-order secular resonance angle,

$$
\theta_{\Omega} \equiv \Omega_{1}-\Omega_{2}=\theta_{i_{1}^{2}} / 2-\theta_{i_{2}^{2}} / 2
$$

about $180^{\circ}$. The latter means that the two orbits are librating about anti-alignment of their lines of nodes. Fig. $1 \mathrm{H}$ shows that the libration amplitude is only a few degrees.

Furthermore, simultaneous libration of $\theta_{e_{1}}, \theta_{e_{2}}$, and $\theta_{\Omega}$ also implies the libration of the resonance angles

$$
\theta_{e_{1} i_{1} i_{2}} \equiv \lambda_{1}-2 \lambda_{2}+\tilde{\omega}_{1} \pm\left(\Omega_{1}-\Omega_{2}\right)=\theta_{e_{1}} \pm \theta_{\Omega}
$$

and

$$
\theta_{e_{2} i_{1} i_{2}} \equiv \lambda_{1}-2 \lambda_{2}+\tilde{\omega}_{2} \pm\left(\Omega_{1}-\Omega_{2}\right)=\theta_{e_{2}} \pm \theta_{\Omega}
$$

The near-perfect anti-alignment of the lines of nodes can be understood in terms of the conservation of that component of the system's angular momentum which lies in the original 

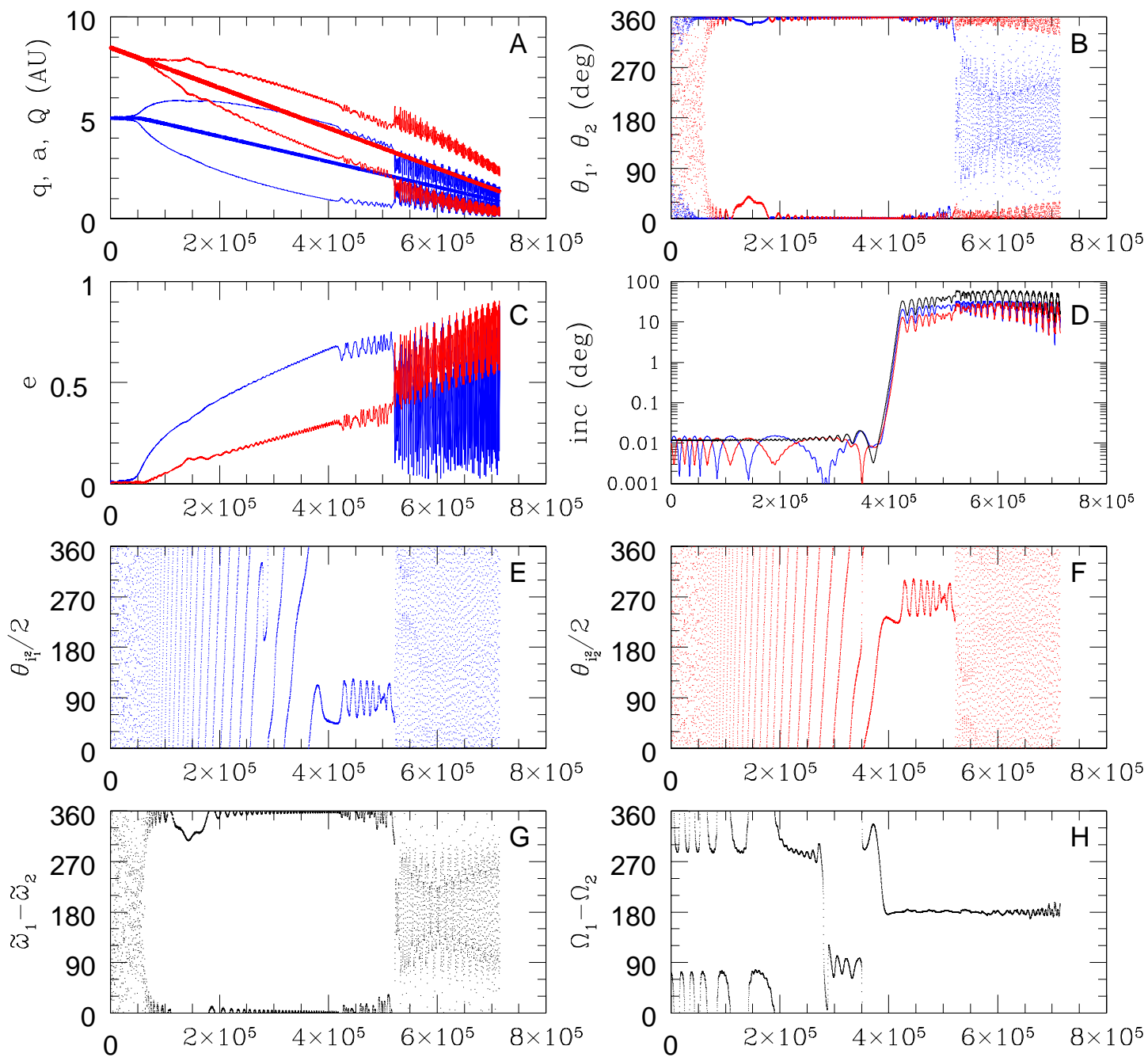

$\mathrm{t}(\mathrm{yrs})$

Fig. 1. - Evolution of the two planets in the baseline run, in which an inward migration rate of $\dot{a}=10^{-5} \mathrm{AU} /$ year is imposed on the outer one. Both planets are one Jupiter mass $\left(1 \mathrm{M}_{J}\right)$; quantities related to the inner (Planet 1) are plotted in blue, and in red for the outer (Planet 2). The panels show: A. Semimajor axis, periastron distance and apastron distance; B. The lowest-order eccentricity-type resonance angles $\theta_{e_{1}}$ (blue) and $\theta_{e_{2}}$ (red); C. Eccentricities of both planets; D. Inclinations of both planets, as well as (black) the mutual inclination; E., F. $\theta_{i_{1}^{2}} / 2$ and $\theta_{i_{2}^{2}} / 2$ where $\theta_{i_{1}^{2}}$ and $\theta_{i_{2}^{2}}$ are the lowest-order inclination-type resonance angles; G. The angle between the planets' longitudes of periastron; H. The angle between the planets' lines of nodes. 

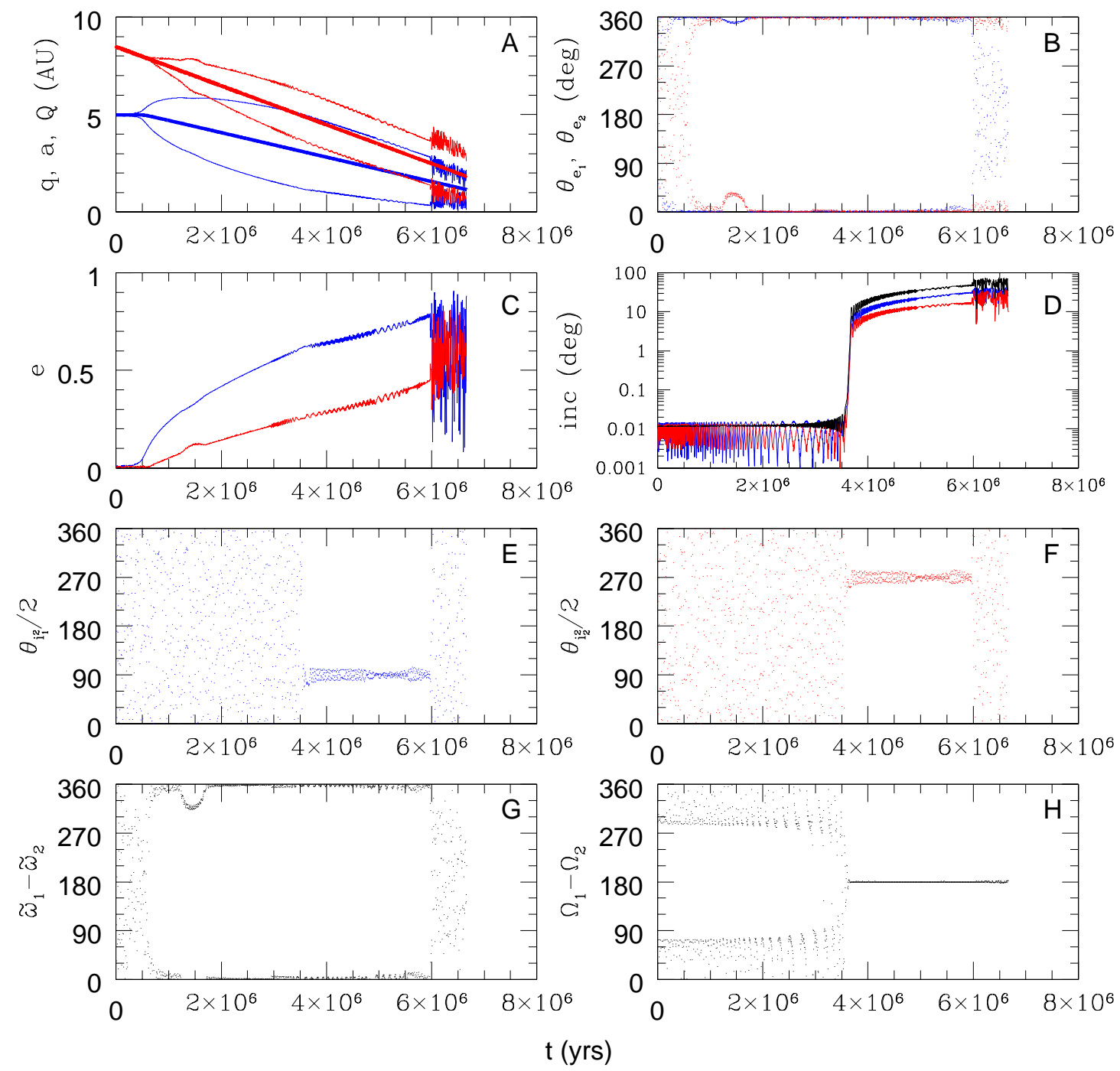

Fig. 2.- Evolution of the planets when the migration rate imposed on the outer planet is $10^{-6} \mathrm{AU} /$ year, one tenth the rate in the baseline run (Fig. 1). All panels are as in Fig. 1. 
orbital plane. Since this is initially very near to zero, the in-plane components of the two orbits' angular momentum vectors have to be pointing in very nearly opposite directions once the inclinations get large, otherwise they cannot sum to (almost) zero. This also means that the relative inclination of the two planets' orbital planes (see 1D) is the sum of their individual inclinations. Thus the relative inclination of the two planets very quickly goes up to $30^{\circ}$.

At $5.2 \times 10^{5}$ years, the system evolves out of both inclination-type resonances. There is an abrupt increase in inclinations at this time, but thereafter, as is to be expected, there is no further net inclination increase. At the same time, the inner planet's eccentricity-type resonance angle, $\theta_{e_{1}}$, switches from libration about $0^{\circ}$ to "near-libration" about $180^{\circ}$ : The angle librates about $180^{\circ}$ for a few cycles, then makes a complete revolution, librates for another few cycles, and so on. From a perturbative analysis point of view, this means that the contribution from the corresponding term in the expansion of the disturbing function continues to be important; its cosine argument $\theta_{e_{1}}$ spends most of its time around $180^{\circ}$ so the term does not average to zero over time. An interesting point is that in a completely planar system, this transition in libration center is inevitably cut short by the system becoming unstable. This happens because the system is trying to evolve from conjunctions at both planets' pericenters, to conjunctions at the outer planet's pericenter and the inner planet's apocenter. Somewhere in between, then, the two bodies will reach a conjunction at the point where their orbits cross, bringing about a very close encounter or even a physical collision (Lee \& Peale 2003, and Lee \& Peale, private communication). However, if a significant

inclination between the two orbits has been attained prior to this transition, as is clearly the case in Fig. 1, this will serve as a protection mechanism against close encounters, allowing the system to stably evolve through the transition in $\theta_{e_{1}}$ 's libration center.

The simulation is stopped at a little after $7 \times 10^{5}$ years, when the inner body comes within $0.1 \mathrm{AU}$ of the star. Other runs with randomly varied initial orbital phases, as well as different intial positions of the planets (though still starting outside the 2:1 mean-motion resonance) produce a similar evolution; in each case the the inclination resonance is encountered after capture into the 2:1 eccentricity resonance, and inclinations grow rapidly thereafter.

\section{Dependence on migration rate and initial inclination}

Another example is shown in Fig. 2. In this case, we reduce the speed at which the disk edge moves inward, $\dot{r}_{\text {accr }}$, by an order of magnitude, which amounts to letting $\alpha=10^{-4}$. The evolution continues to be very similar to that shown in Fig. 1, scaled up by a factor of ten in time. However, in slowing the migration rate we have also made the migration more 
adiabatic; that is, we have reduced the amount of migration during one libration period of the resonance(s). Thus, it is to be expected that the dynamics change by more than a simple rescaling in time. One difference apparent from Fig. 2 is that libration amplitudes are reduced; the average values of $e$ and $i$, however, are very close to those in the faster migration. Another difference is that the times at which the inclination resonances are entered and exited do not scale exactly with the slower migration speed.

We also probe the dependence of the system's evolution on the initial mutual inclination of the two orbits. Up to now, we have made this $\sim 0.01^{\circ}$. We perform a run where the mutual inclination is decreased to $\sim 0.0005^{\circ}$, and another where it is increased to $1^{\circ}$. The resulting inclination evolution of the two planets is plotted in Fig. 3, together with the baseline case of the previous section. The smaller initial inclination causes very little change in the time of onset of the inclination excitation, the timescale over which the inclination is subsequently excited $\left(\sim 2 \times 10^{4}\right.$ years), or the value to which the mutual inclination is subsequently excited $\left(\sim 30^{\circ}\right)$. The larger initial inclination causes a slightly earlier onset of inclination growth, by an amount $\Delta t / t \sim 0.05$. Evidently, therefore, entry into the inclination resonance is not strongly influenced by the actual value of the initial mutual inclination, as long as it is small, $\lesssim 1^{\circ}$.

\section{Varying the mass ratio}

We next investigate the effect of making the mass ratio of the two planets, $M_{1} / M_{2}$, differ from unity. We find that when $M_{1} / M_{2} \lesssim 2$, the inclination resonance is no longer reached. This is because onset of the resonance requires the inner body to have a large eccentricity; however, when there is a large disparity in the masses of the two planets, the eccentricity of the more massive one will not grow very much. Fig. 4 shows an example in which $M_{1} / M_{2}=3$. Although the outer body's eccentricity has reached 0.85 by the end of the run, that of the inner body does not get above 0.2 , and the inclination resonance is avoided. We defer a detailed investigation of the conditions for onset of the inclination resonance to future work. For the time being, it is useful to recall that the simultaneous libration of a body in the 2:1 eccentricity-type resonance and the 4:2 inclination-type resonance requires that its

longitude of pericenter precession rate, $\dot{\tilde{\omega}}$, be on average equal to the precession rate of its line of nodes, $\dot{\Omega}$ (as in a Kozai resonance, e.g., Murray \& Dermott 1999). Both of these rates change as the eccentricity increases; for a body already in the eccentricity-type resonance, we expect that the inclination-type resonance sets in when the rates become equal.

When the inner body is smaller, the inclination resonance is still entered for much more disparate masses. Fig. 5 shows the evolution when the outer planet is Jupiter-mass and the 

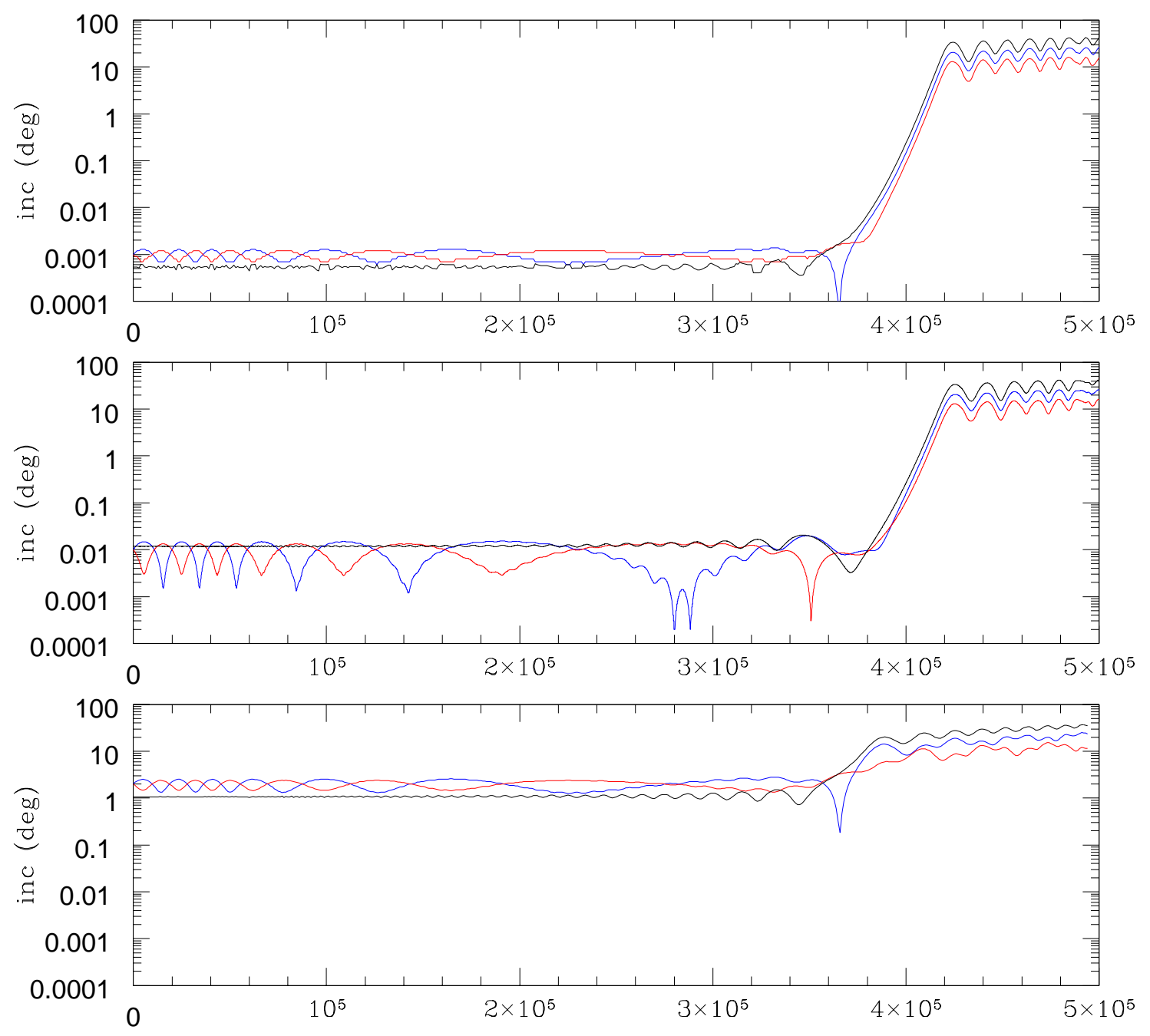

Fig. 3.- Evolution of the planets' inclinations for differing initial mutual inclinations of $<0.001^{\circ}$ (top), $0.01^{\circ}$ (middle; the baseline case), and $1^{\circ}$ (bottom) 
inner is Earth-mass, making $M_{1} / M_{2} \sim 1 / 300$. Though the behaviour is qualitatively similar to the equal-mass case (Fig. 1), there are some obvious differences: As is to be expected from conservation of momentum, the small planet suffers far more inclination excitation than the large one. Also, there is a delay of $\sim 5 \times 10^{4}$ years between the onset of libration in $\theta_{i_{1}^{2}}$ and $\theta_{i_{2}^{2}}$, and the inclination growth, which begins with the libration of $\theta_{i_{1}^{2}}$, is not as fast as in the equal-mass case.

We perform additional simulations in which $M_{1} / M_{2} \sim 10^{-8}$, making it effectively a test particle (with $M_{2}$ still being Jupiter mass, $M_{1}$ is now comparable to the largest asteroids). Yu \& Tremaine (2001) find that a test particle in a 2:1 resonance with an inward-migrating massive body, initially inclined relative to the massive body by of order $1^{\circ}$, can have its inclination excited all the way up to $90^{\circ}$ and then be released from the 2:1 resonance altogether. When we use a similar initial inclination, we get capture into inclination resonance and excitation of the small body's inclination. We only follow the evolution of the system until the inner body's pericenter drops below $0.1 \mathrm{AU}$; at this point the body is still trapped in both the eccentricity and inclination resonance, and its inclination, which is still growing, has reached $\sim 70^{\circ}$. It is now only $\theta_{e_{1}}$ and $\theta_{i_{1}^{1}}$, not $\theta_{e_{2}}$ and $\theta_{i_{2}^{2}}$, which librate. With an initial mutual inclination of $0.01^{\circ}$, however, it is possible for the small body to quickly pass through the inclination resonance, "librating" for less than a full cycle before leaving it again, while having its inclination excited to only $\sim 1^{\circ}$. Though Yu \& Tremaine (2001) do not check for libration in inclination-type resonances, the similarity in evolution of eccentricity and inclination between our simulations and their Fig. 5 leads us to believe that the behaviour they see is the $M_{1} / M_{2} \rightarrow 0$ limit of the mechanism we explore here. In any case, we do not investigate the behaviour at such extreme mass ratios in more detail in the present work; if we are to consider pairs of giant planets, the possible masses range from $10^{1}$ to $10^{3} \mathrm{M}_{\oplus}$, so the smallest possible ratio is of order $M_{1} / M_{2} \sim 10^{-2}$, as in the case pictured in Fig. 5.

\section{Damping of eccentricities}

Next, we consider the possibility that planet-disk interactions may damp planetary eccentricities. Following Lee \& Peale (2002), we make the relative eccentricity damping rate proportional to the relative applied semimajor axis decay rate for the outer planet:

$$
\frac{\dot{e}_{2}}{e_{2}}=S \frac{\dot{a}_{2}^{\text {appl }}}{a_{2}} .
$$

The applied decay rate, $\dot{a}_{2}^{\text {appl }}$, is the rate at which the outer planet by itself would migrate under the action of the applied torque. However, because the outer planet has to push along 

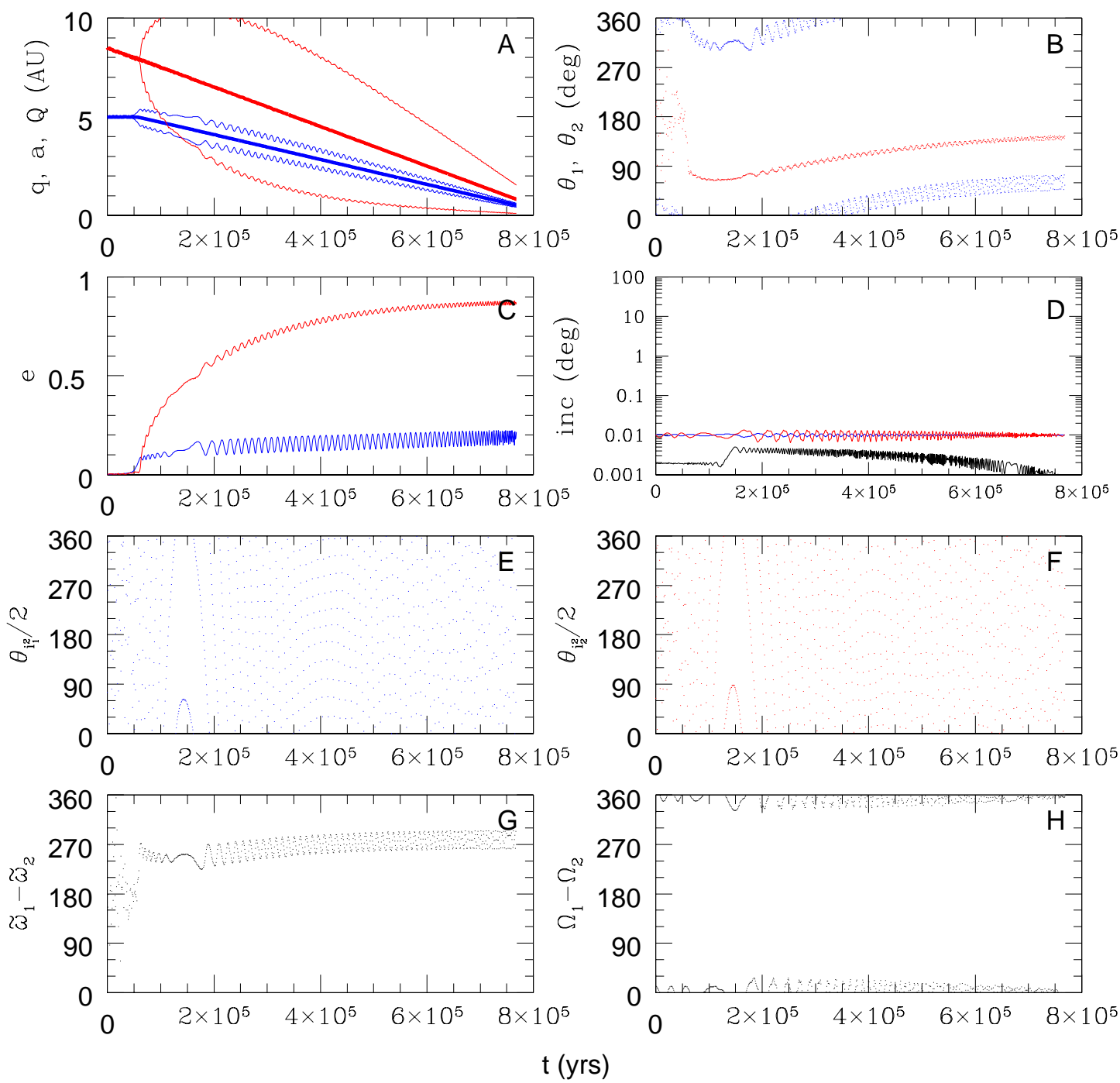

Fig. 4.- Evolution of the planets in the case where the inner is Jupiter-mass, and the outer is one third that, i.e., Saturn-mass (Section 6). The panels are as in Fig. 1. 

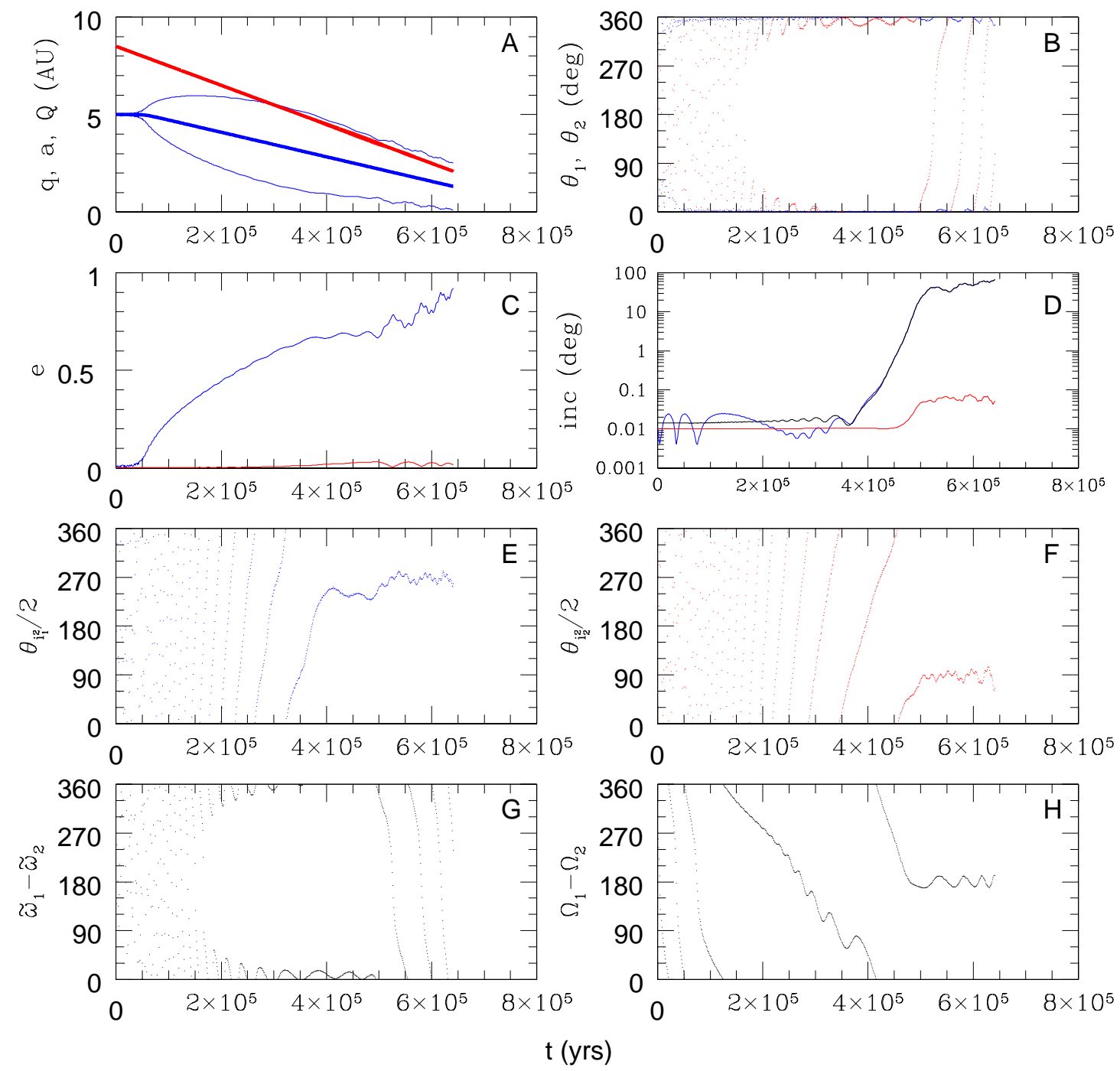

Fig. 5.- Evolution of the planets in the case where the outer planet is Jupiter-mass, and the inner $\sim 1 / 300$ Jupiter mass, i.e., Earth-mass (Section 6). The panels are as in Fig. 1. 
the resonantly-locked inner planet, its actual migration rate, $\dot{a}_{2}$, is less than $\dot{a}_{2}^{\text {appl }}$. Recall that torque is applied in such a way as to keep $\dot{a}_{2}$ (nearly) constant (Eq. 3).

This eccentricity damping rate is applied to the outer planet only; the inner is, again, assumed to not be interacting with the disk directly. We perform simulations with $S=2$ and $S=5$. Results are shown in Fig. 6. In the $S=2$ case, the inclination resonance is entered later due to the slower eccentricity growth, and the maximum mutual inclination reached is less than $20^{\circ}$. When $S=5$, the planets' eccentricities reach equilibrium values before the inclination resonance is attained, and the system thus remains coplanar.

One effect we have not modeled is direct damping of the planets' inclinations by the disk. Lubow \& Ogilvie (2001) find that such damping should occur when $\alpha \gtrsim 10^{-3}$, and proceed on a timescale of order $10^{5}$ years. Thus, our baseline runs, with $\alpha=10^{-3}$, are at the limit of where damping is expected to occur. Also, the inclination damping timescale is long compared to the timescale of inclination growth when the inclination resonances are first encountered, which is only a few $\times 10^{4}$ years. Thus, damping of inclinations by the gas disk likely has little effect on inclinations excited by resonances among planets.

\section{The disk potential}

Thus far we have only included the effect of the gas disk in the form of a forced migration experienced by the outer planet. However, the disk may influence the dynamics of the planets in other important ways. Specifically, the gravitational force from a disk will modify the planets' nodal and apsidal precession rates, $\dot{\Omega}$ and $\dot{\tilde{\omega}}$. This can change the locations and relative spacings of the mean-motion resonances, and so might change the nature of the system's resonant evolution. Therefore, it is important to incorporate the potential of the disk into our analysis. We assume that the "disk" is a thin annulus of surface density $\Sigma(r)$ located in the $z=0$ plane, with inner radius $r_{1}$ and outer radius $r_{2}$, where $r$ is the stellocentric distance. The potential due to the disk, as felt at a radius $R$ and height $z$, is

$$
\Phi(R, z)=-\int_{r_{1}}^{r_{2}} \int_{0}^{2 \pi} \frac{G \Sigma(r) r d \phi d r}{\sqrt{R^{2}+r^{2}+z^{2}-2 r R \cos \phi}}
$$

and the corresponding force is $F_{R}(R, z)=-\partial \Phi / \partial R, F_{z}(R, z)=-\partial \Phi / \partial z$. To obtain the force due to an outer disk on a body, we can expand the numerator in powers of $R / r$ and $z / r$, then perform the integration. Assuming a surface density of the form

$$
\Sigma(r)=\Sigma_{0} r^{-3 / 2}
$$

i.e., having the density profile of the standard Hayashi (1981) nebula model, we obtain 

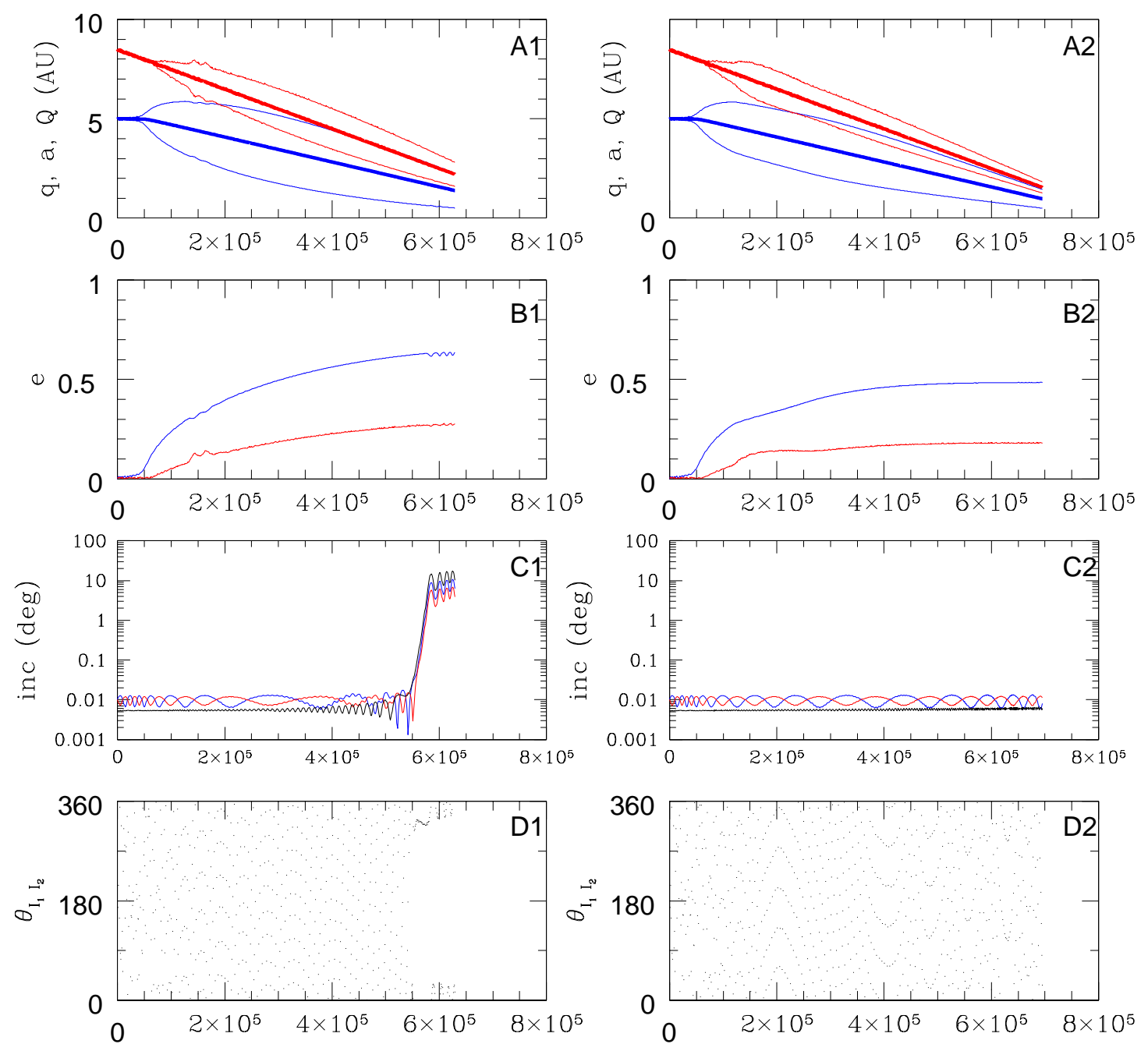

Fig. 6.- Evolution of the two-planet system when the eccentricity of the outer is damped. The left set of panels show the case where the relative damping rate, $\dot{e}_{2} / e_{2}$, is twice the applied relative semimajor axis decay rate, $\dot{a}_{2}^{\text {appl }} / a_{2}$; the right set show the case where the relative damping rate is $5 \times \dot{a}_{2}^{\text {appl }} / a_{2}$. The individual panels show: A. Semimajor axis, periastron and apastron distance; B. Eccentricities; C. Inclinations; D. The mixed inclination-type resonance angle $\theta_{i_{1} i_{2}}$ 


$$
F_{R}(R, z)=\left.\frac{G \Sigma_{0} \pi}{r^{3 / 2}}\left(-\frac{2}{5}(R / r)+(R / r)^{3}-\frac{2}{3}(R / r)(z / r)^{2}+\ldots\right)\right|_{r=r_{1}} ^{r=r_{2}}
$$

and

$$
F_{z}(R, z)=\left.\frac{G \Sigma_{0} \pi}{r^{3 / 2}}\left(\frac{4}{5}(z / r)+(R / r)^{2}(z / r)-\frac{2}{3}(z / r)^{3}+\ldots\right)\right|_{r=r_{1}} ^{r=r_{2}} .
$$

We performed a simulation with a disk surface density of 5 times $\Sigma_{\min }$, where

$$
\Sigma_{\text {min }}=1.7 \times 10^{3}(r / 1 \mathrm{AU})^{-3 / 2} \mathrm{~g} / \mathrm{cm}^{2}
$$

is the Hayashi minimum-mass surface density. The inner radius of the disk is made to be 20 Hill radii of the outer planet beyond $r_{\text {disk }}$ at all times. The Hill (or Roche) radius of a planet of mass $M$ orbiting at a distance $r$ from a primary of mass $M_{*}$ is $r_{H}=\left(M / 3 M_{*}\right)^{1 / 3} r$. The actual width of a planet-induced gap is likely significantly smaller, between 3 and 4 Hill radii (e.g., Bryden, Różyczka, Lin, \& Bodenheimer 2000). However, since the migrating planets tend to acquire large eccentricities, we endeavour to keep the disk edge far enough away that it lies well beyond the planets' apocenters at all times, thus keeping the expansion of the potential valid. The response of a disk to an eccentric gap-opening planet is a complicated problem which has thus far not been investigated (e.g., Lin et al. 2000), so to avoid having to make arbitrary assumptions, we restrict ourselves to modeling the gravitational interaction of the planets with the more distant part of the disk.

The evolution of the system is shown in Fig. 7. Comparing to Fig. 1, one can see that the evolution is quite similar to the diskless case. Despite the additional radially-varying apsidal precession induced by the disk potential, the two planets again become apsidally aligned upon encountering the 2:1 resonance. Also, despite the change in the vertical frequency, the inclination resonance sets in at nearly the same time as in Fig. 1. One noticeable difference is that the angle between the planets' lines of nodes, $\Omega_{1}-\Omega_{2}$, has a larger amplitude of libration about $180^{\circ}$, and in fact circulates briefly several times. This is possible because, with the addition of the disk potential, the two-planet system no longer conserves the component of its angular momentum which lies in the disk plane.

\section{Additional planets}

Finally, we investigate the effect that additional smaller planets have on migration in resonance. We add four bodies, each $15 \mathrm{M}_{\oplus}$ in mass (i.e., Uranian or "ice giant" size), interior to the inner of the two Jupiter-mass bodies. The bodies are separated by between 10 and 15 mutual Hill radii, so as to form a relatively stable system. The innermost initially 

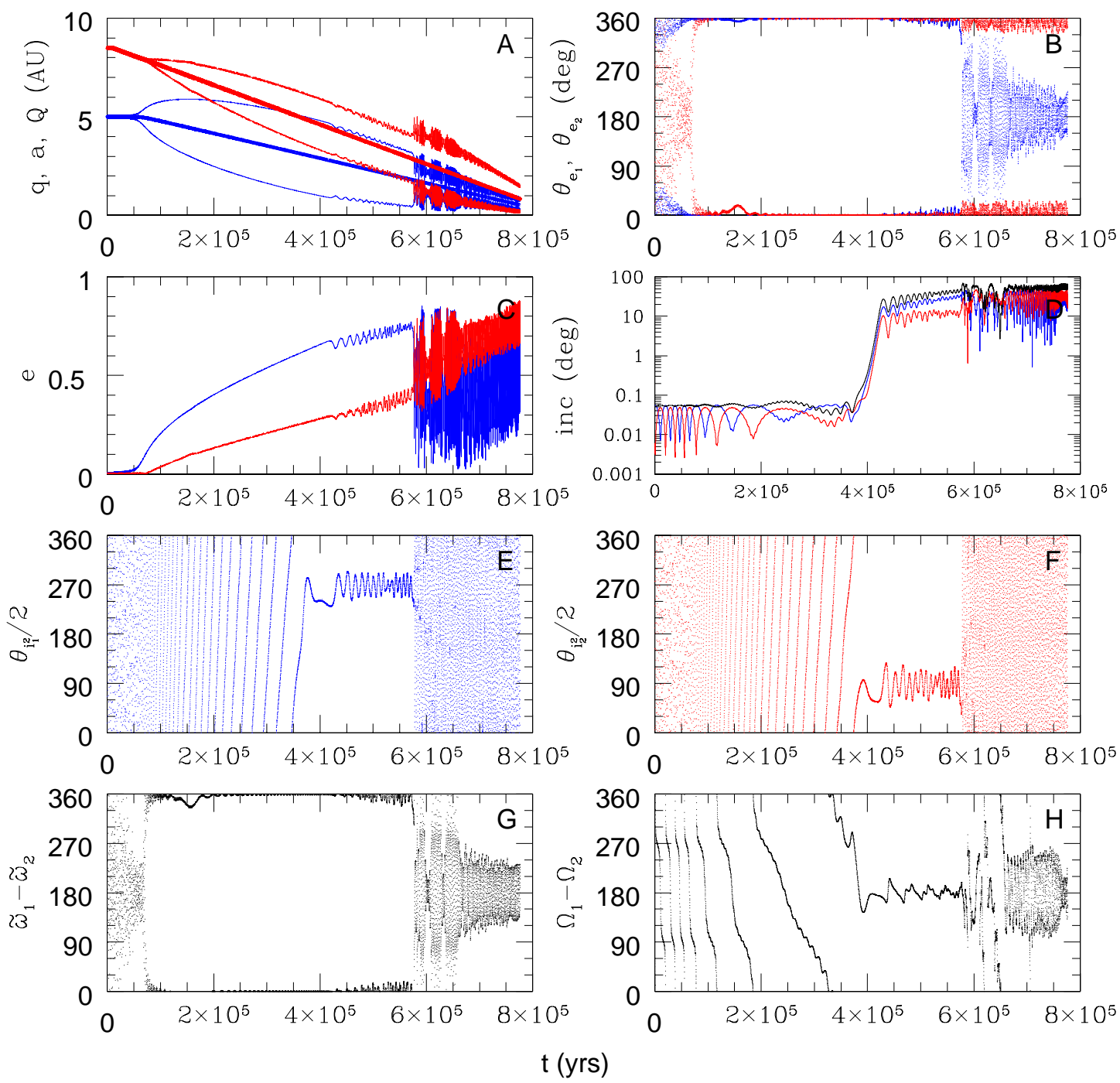

Fig. 7.- Evolution of the two-planet system with the addition of the potential of a $5 \times$ minimum-mass outer disk. Panels are as in Fig. 1. 
has a semimajor axis around $1 \mathrm{AU}$, while the outermost is at $3 \mathrm{AU}$, just short of the interior 2:1 resonance of the Jovian body at $5 \mathrm{AU}$. We do not argue that it is likely for a planetary system's inner region to contain so many large bodies at any time in its evolution. In-situ formation alone is, at any rate, unlikely to produce such massive bodies in this region (e.g., Lissauer 1987; Thommes, Duncan, \& Levison 2003). Rather, our intent is to put into the simulation about as much mass in sub-gas giant bodies as will stably fit in the region. In this way, we obtain an idea of the upper limit (apart from stochastic close approaches) on the perturbations that the inner part of the planetary system could exert on the resonant migration of the two gas giants.

Fig. 8 shows the resulting orbital evolution, including semimajor axes of the Uranianmass bodies, in one of a set of eight simulations performed. The last of these extra bodies is eliminated at $3 \times 10^{5}$ years. All are removed by crossing the inner simulation boundary, which in these runs is at $0.5 \mathrm{AU}$. Comparing to Fig. 1, there are some key differences between the orbital evolution of the two gas giants here and in the baseline simulation. Here, the onset of the inclination resonance, shown by the transition of $\theta_{i_{1}^{2}}$ and $\theta_{I_{2}^{2}}$ from circulation to libration, takes place about $2 \times 10^{5}$ years later than in the baseline simulation. However, the gas giants already acquire significant inclinations before this happens. The growth of the inclinations begins at about the same time as $\theta_{\Omega}$, the difference between the lines of nodes, begins to librate about $180^{\circ}$. This means that, although the two planets are not in the pure inclination-type resonances, they are (since $\theta_{e_{1}}$ and $\theta_{e_{2}}$ are librating at the same time) in the mixed eccentricity-inclination resonances involving the libration of $\theta_{e_{1} i_{1} i_{2}}$ and $\theta_{e_{2} i_{1} i_{2}}$.

The pure inclination-type resonances do not set in until after the last Uranian planet is eliminated, and final inclinations are comparable to those reached at the same time in the baseline run. The transition from libration about $0^{\circ}$ to libration about $180^{\circ}$ of one of the eccentricity-type resonances seen in Fig. 1 does not occur here, simply because the inner boundary in this run is at $0.5 \mathrm{AU}$ instead of $0.1 \mathrm{AU}$ (to allow a longer timestep and thus speed the integration), and therefore the inner Jovian planet is eliminated before its libration center can switch.

With this many bodies, the system becomes strongly stochastic. Of the eight runs performed, six evolve in a manner qualitatively similar to the one shown in Fig. 8: The gas giants undergo resonant capture and evolve inward together, pushing the interior planets ahead of themselves. In all of these cases, significant resonant excitation of the giant planets' inclinations occurs, though in some cases a 3:1 rather than a 2:1 commensurability is involved. In the two remaining runs, the gas giants undergo a physical collision. This happens because close encounters with the smaller planets can exert a strong enough perturbation on the large planets to temporarily knock them out of resonance with each other. The large planets' orbits 

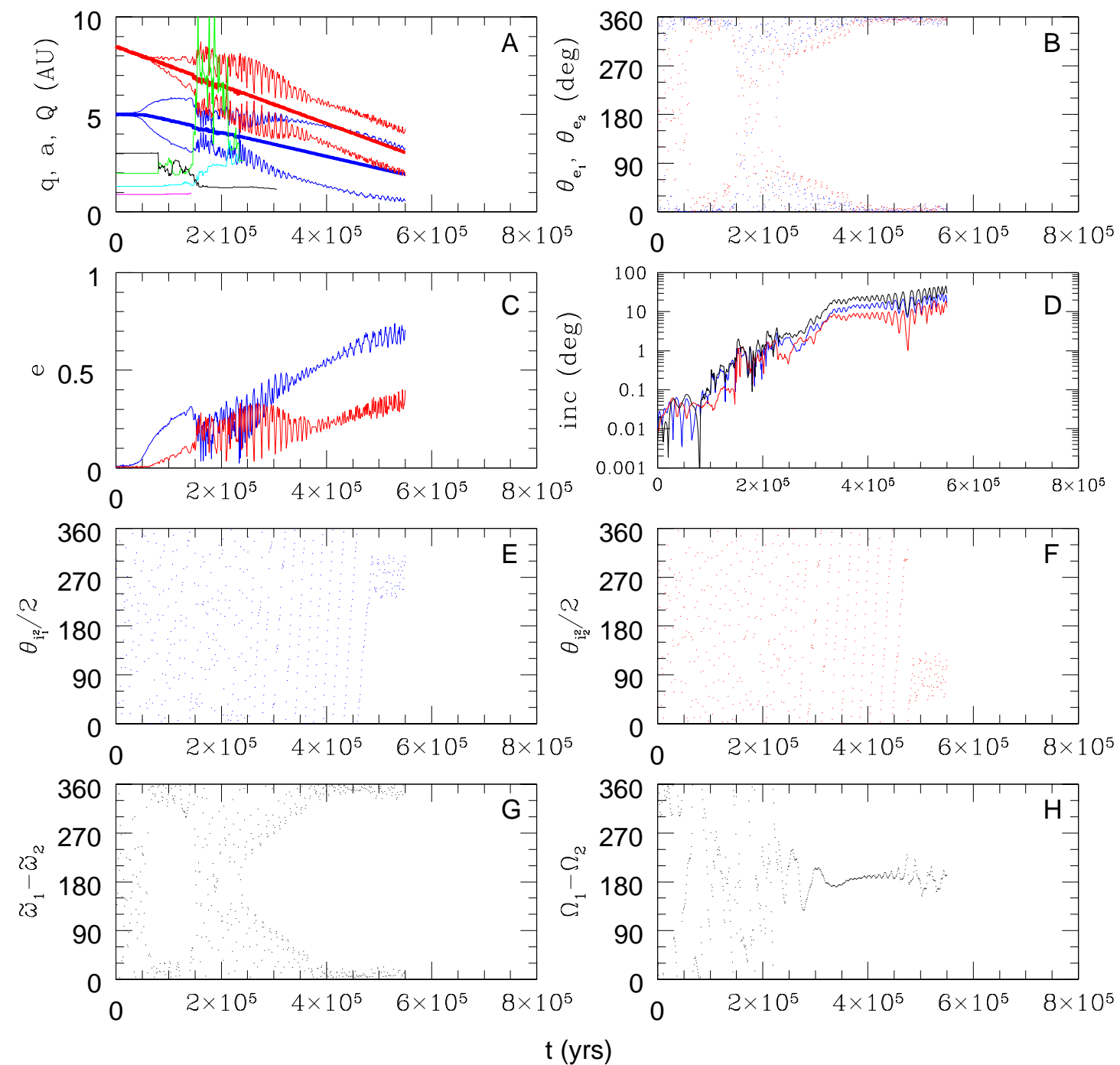

Fig. 8.- An example of the evolution of the system with four $15 \mathrm{M}_{\oplus}$ (Uranian mass) planets added interior to 3 AU. Panels are as in Fig. 1, and additionally the semimajor axes of the Uranian planets are shown (in black, green, cyan and magenta) in Panel A. The last of them is eliminated at about $3 \times 10^{5}$ years. 
1
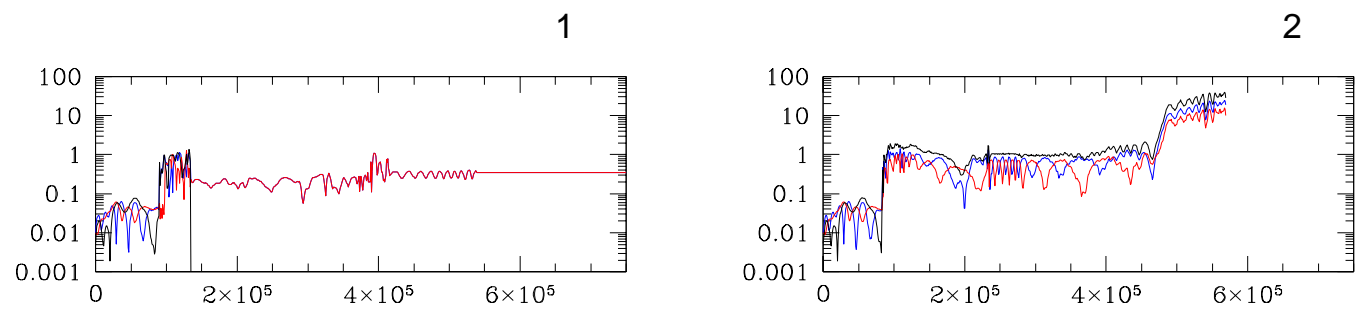

3
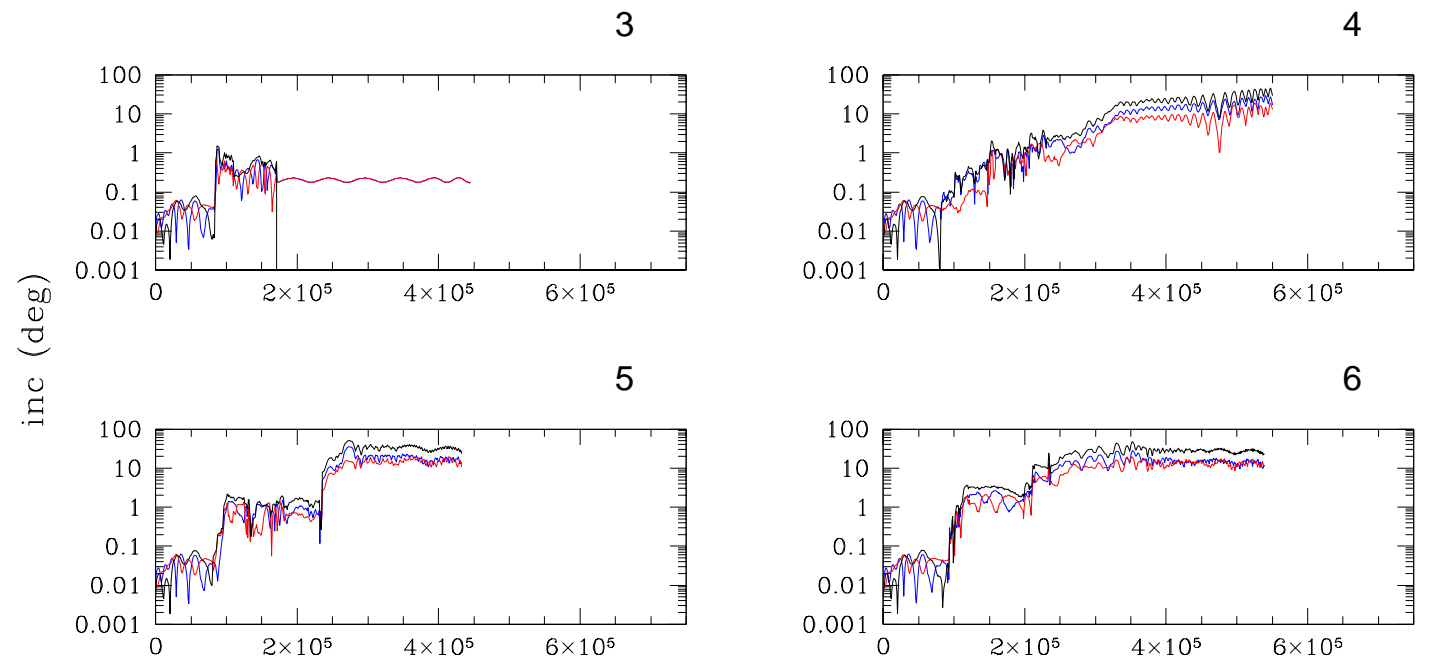

7
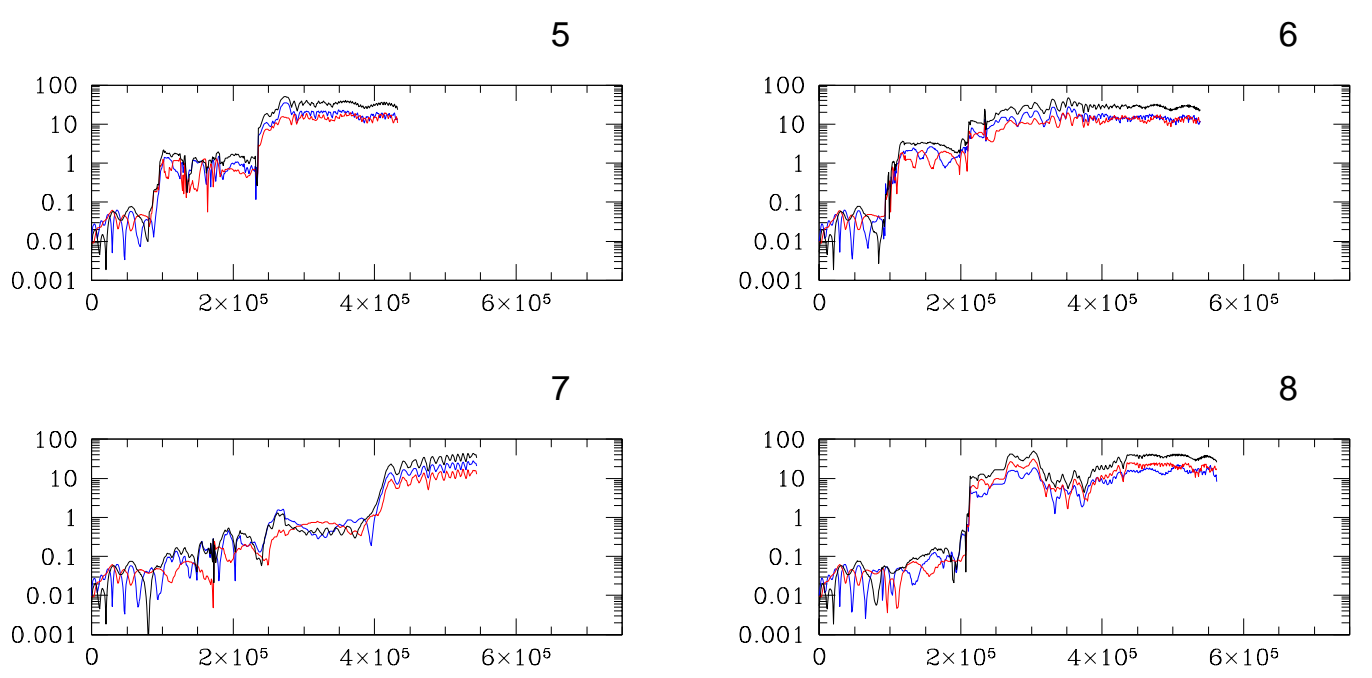

Fig. 9.- The full set of eight runs performed with four inner Uranian planets, showing the evolution of the two Jupiter-mass planets' inclinations in each case. In runs 1 and 3, the two Jovian planets merge before the inclination resonances are encountered. 
already cross by this time, so while their resonant lock is removed, they are free to undergo close encounters with each other.

Fig. 9 shows the evolution of the inclinations in each of the eight runs. Runs 1 and 3 are the ones where the gas giants collide, in both cases before significant inclinations are excited. In all of the other six runs, relative inclinations in excess of $30^{\circ}$ arise.

\section{Discussion}

We have shown that two planets migrating in resonance can undergo significant excitation of their inclinations under a wide range of conditions. The excitation mechanism is libration in the lowest order (order $i^{2}$ ) inclination-type mean-motion resonances. Even though convergent migration initially leads to capture into a first-order eccentricity-type resonance, at sufficiently large eccentricity, the two bodies can librate simultaneously in both types. Previous work has also suggested the possiblity of high inclinations arising during the formation of planetary systems (Levison, Lissauer, \& Duncan 1998; Yu \& Tremaine 2001). In the former case, the inclination excitation mechanism considered was stochastic close encounters among planets. However, since these typically eventually lead to ejections and mergers, one would expect few long-lived non-coplanar systems of closely-spaced planets to be produced in this way. In the latter case, the authors showed that massless test particles resonantly trapped by an inward-migrating planet can eventually be released from resonance on polar and even retrograde orbits; this suggests the possibility of non-coplanarity between giant planets and much smaller bodies. We think it is likely that the effect seen by the authors is actually the $M_{1} / M_{2} \rightarrow 0$ limit of the mechanism describe herein (see Section 6).

Resonant inclination excitation is quite robust against external perturbations. Adding the potential of a fairly massive ( 5 times minimum mass) outer gas disk into the simulation does not prevent the onset of the inclination resonance. Neither, in most cases, does the addition of a massive inner planetary system. However, it appears that the inclination resonances cannot be encountered until the eccentricity of the inner planet gets quite high, $e_{1} \gtrsim 0.6$. Thus, damping of the eccentricity can prevent excitation of the inclinations. Given equal-mass bodies in a 2:1 resonance, we find the threshold relative damping rate for the outer body, $\dot{e}_{2} / e_{2}$, is between 2 and 5 times the relative applied migration rate, $\dot{a}_{2}^{\text {appl }} / a_{2}$ (the inner planet is assumed not to interact with the disk). Also, if the mass ratio $M_{1} / M_{2}$ is larger than about 2 , the inner body undergoes very little eccentricity excitation and the inclination resonances are avoided.

There is no obvious reason why having two adjacent companions with $M_{1} / M_{2}<2$ should 
be an uncommon feature of planetary systems. In fact, the majority of discovered multiplanet systems, and both of the systems in 2:1 resonance (see below), fulfill this condition at least with their projected masses, $M \sin i$. Our own Solar System, it should be pointed out, does not $\left(M_{\text {Jupiter }} \approx 3 M_{\text {Saturn }}\right)$. Less clear, though, is the issue of the disk's effect on planet eccentricities. There is not even a consensus on whether the disk-induced eccentricity rate of change, $\dot{e}_{\text {disk }}$, is positive or negative. Goldreich \& Sari (2003) analytically show that the former ought to be the case. However, numerical simulations have tended to support the latter; for example, Nelson et al. (2000) perform simulations of gap-opening planets with three different hydrodynamic codes and find $\dot{e}_{\text {disk }}$ to be negative in each case. Also, in the simulations of Papaloizou, Nelson, \& Masset (2001), disk interactions do not cause eccentricity growth until the planetary mass exceeds $\sim 20 \mathrm{M}_{J}$. The problem is that the competing effects of eccentricity damping at corotation resonances and eccentricity pumping at Lindblad resonances are very nearly of equal strength. Whether one or the other dominates depends sensitively on the details of the disk's surface density profile in the vicinity of the planet, which is a difficult issue to handle either numerically or analytically. In any case, an eccentricity damping timescale of the same order as the orbital decay timescale or longer, which we find is required to permit capture into inclination resonances, is entirely plausible. A related question is whether the disk might impose some maximum eccentricity on embedded planets. As previously mentioned, the standoff distance between a disk and a gap-opening planet's orbit is between 3 and 4 Hill radii in numerical simulations (e.g., Bryden et al. 2000). Assuming this distance is independent of planetary eccentricity, a Jupiter-mass planet will run into the inner disk edge at apocenter when its eccentricity is $\sim 0.2-0.3$, which may lead to increased damping. In all of the simulations performed here (except for the one shown in Fig. 4, but there the inclination resonance was avoided anyway), the outer planet's eccentricity is $\sim 0.3$ or less at the time the inclination resonance is entered. Thus, if such a gap-edge cutoff exists, a pair of resonant planets still has a good chance of entering the inclination resonance before suffering significant eccentricity damping.

We find that once capture into the inclination resonances sets in, the inclinations of the planets can rapidly grow to $\sim 15^{\circ}$ relative to their plane of origin. Since the lines of nodes of the two planets' orbits are anti-aligned after entering the inclination resonance, the relative inclination is the sum of each orbit's inclination, and grows to $\sim 30^{\circ}$. Later, when the system undergoes large coupled oscillations in eccentricity and inclination, mutual inclinations can get as high as $\sim 60^{\circ}$ (Fig. 1). Thus, a planetary system which enters inclination resonance during its evolution may end up in a highly non-coplanar configuration. This is an important possiblity to consider in dynamical investigations of exoplanetary systems; in fact, even the inclination relative to our line of sight, and thus the sin $i$ scale factor for the planetary masses, may differ significantly among individual members of multi-planet systems. 
Among the discovered exoplanetary systems, the best candidates for having mutuallyinclined orbits due to the mechanism described here are those in which the companions are (thought to be) in a mean-motion resonance. In the case of the GJ 876 system, Rivera \& Lissauer (2001) perform dynamical fits allowing for the possiblity of mutual inclinations. Most of the systems they find which both provide good fits to the radial velocity data and long-term stability have mutual inclinations of $12^{\circ}$ or less. They also find one (not necessarily the only) stable, locally best-fit (in the sense of a local $\chi^{2}$ minimum) region at a very high mutual inclination of $77^{\circ}$. Leaving aside this extreme case, since none of the runs performed here result in such a high mutual inclination for any significant length of time, one might thus conclude that GJ 876 could have undergone resonant inclination excitation, provided some mechanism-perhaps eccentricity damping (see Fig. 6), or direct damping of inclinations by the disk, which we have not considered - kept inclinations from getting very high. Also, the outer companion's $M \sin i$ is about 3.4 times that of the inner, so if the outer planet is oriented edge-on to our line of sight (see below), the condition $M_{1} / M_{2}<2$ is fulfilled unless the inclination between the two orbits is $\gtrsim 80^{\circ}$. However, the system's relatively low eccentricities, $\lesssim 0.3$ for the inner planet, and $\lesssim 0.1$ for the outer (Marcy et al. 2001; Laughlin \& Chambers 2001), argue against resonant inclination excitation. Unless the eccentricities were significantly higher than this in the past, it is unlikely that the inclination resonance would ever have been reached.

The two companions of HD 82943 are also thought to be in a 2:1 resonance, and these have inferred eccentricities of 0.41 (outer) and 0.54 (inner) (as announced at an ESO press release, April 4, 2001; see http://obswww.unige.ch/ udry/planet/hd82943syst.html). Also, the outer companion's $M \sin i$ is more than twice that of the inner. This pair of planets may therefore provide a more likely setting for resonant inclination excitation.

The actual mutual inclinations in multiple-planet systems should eventually be revealed by astrometric measurements. In fact, the first and thus far only astrometrically-measured orbit is that of the outer companion of GJ 876 (Benedict et al. 2002), showing its orbit to be oriented nearly edge-on to the line of sight, and thus determining its mass to be about $1.9 \mathrm{M}_{J}$. The smaller semimajor axis and the (very likely) smaller mass of the inner companion, GJ 876c, will make it more difficult to detect astrometrically; if coplanar with GJ $876 \mathrm{~b}$, it should produce a perturbation in the star's position around 0.2 that produced by the outer, or about 0.05 milliarcseconds. Such astrometric precision is attainable in the forseeable future; certainly, it lies well within the planned microarcsecond precision of the Space Interferometry Mission (SIM).

A mechanism for tilting planetary orbits relative to the orientation of the original protoplanetary disk is particularly interesting in the context of the $\beta$ Pictoris dust disk (Kalas \& 
Jewitt 1995). The disk, which is oriented nearly edge-on to the line of sight, displays a warp out to a distance of about $50 \mathrm{AU}$; the disk inside this radius is inclined about $3^{\circ}$ relative to the outer disk. Mouillet et al. (1997) modeled the warp as arising from the influence of a single unseen planet, with a mass between $10^{-2}$ and $10^{-5}$ that of the central star, with a semimajor axis between 1 and $20 \mathrm{AU}$, and inclined 3 to $5^{\circ}$ to the midplane of the outer disk. As long as the inclination resonance is actually reached, the simulations we perform here indicate that such inclinations could easily arise. Additional structure revealed in recent observations of $\beta$ Pic seems even more suggestive: Wahhaj et al. (2003) conclude from their observations that the disk in fact contains multiple warps interior to $100 \mathrm{AU}$, being made up of four inclined rings which they denote A, B, C and D, centered at roughly 14, 28, 52 and $82 \mathrm{AU}$ respectively. They estimate that these these rings are inclined at about $-32^{\circ},+25^{\circ},-2^{\circ}$ and $+2^{\circ}$ respectively, relative to the outer disk. They point out that the ring radii suggest low-order mean-motion commensurabilities: $3: 1$ between $\mathrm{A}$ and $\mathrm{B}$, and 2:1 between $\mathrm{C}$ and $\mathrm{D}$. There is also the possiblility of a higher-order commensurability, 7:3, between B and C. Wahhaj et al. (2003) suggest that these rings are associated with planets. Their observations appear to fit well with a scenario like the one we explore here, in which planets acquire large inclinations relative to the original plane of the protoplanetary disk by migrating in resonance with each other. Particularly intriguing are the alternating positivenegative inclinations of adjacent rings, mimicing the nodally anti-aligned configurations that the planets take on in our simulations once they acquire significant inclinations.

Thus far, we have not considered the ultimate fate of our inward-migrating planets. The simulations are simply stopped once either planet crosses the inner simulation boundary $(0.5$ or $0.1 \mathrm{AU})$. However, the configuration we are ultimately left with is sensitively dependent on how the migration "end game" actually plays out. If migration keeps going down to very near the star, one or both planets will be lost. Alternatively, it is possible that the gas disk could dissipate sometime after resonant capture but before the planets are driven into the star, though this would require a coincidence in timing. In the absence of other effects that outlive the gas, such as interaction with a massive debris disk, interaction with other planets, and for bodies with very small periastron distances, tidal interaction with the primary, the mean inclinations (and eccentricities) should remain at their values at the time migration ceases. Fig. 10 shows an example in which we (abruptly) turn off the induced migration of the outer planet at $6 \times 10^{5}$ years, shortly after the system has entered the inclination resonances.

The longer the migration timescale (i.e., the lower the disk viscosity), the more plausible such a coincidence becomes. Another possibility to consider is that migration may under some circumstances be outward, for instance if a resonant pair of planets does not form a clean gap and mass flows inward across the gap, as demonstrated by Masset \& Snellgrove 

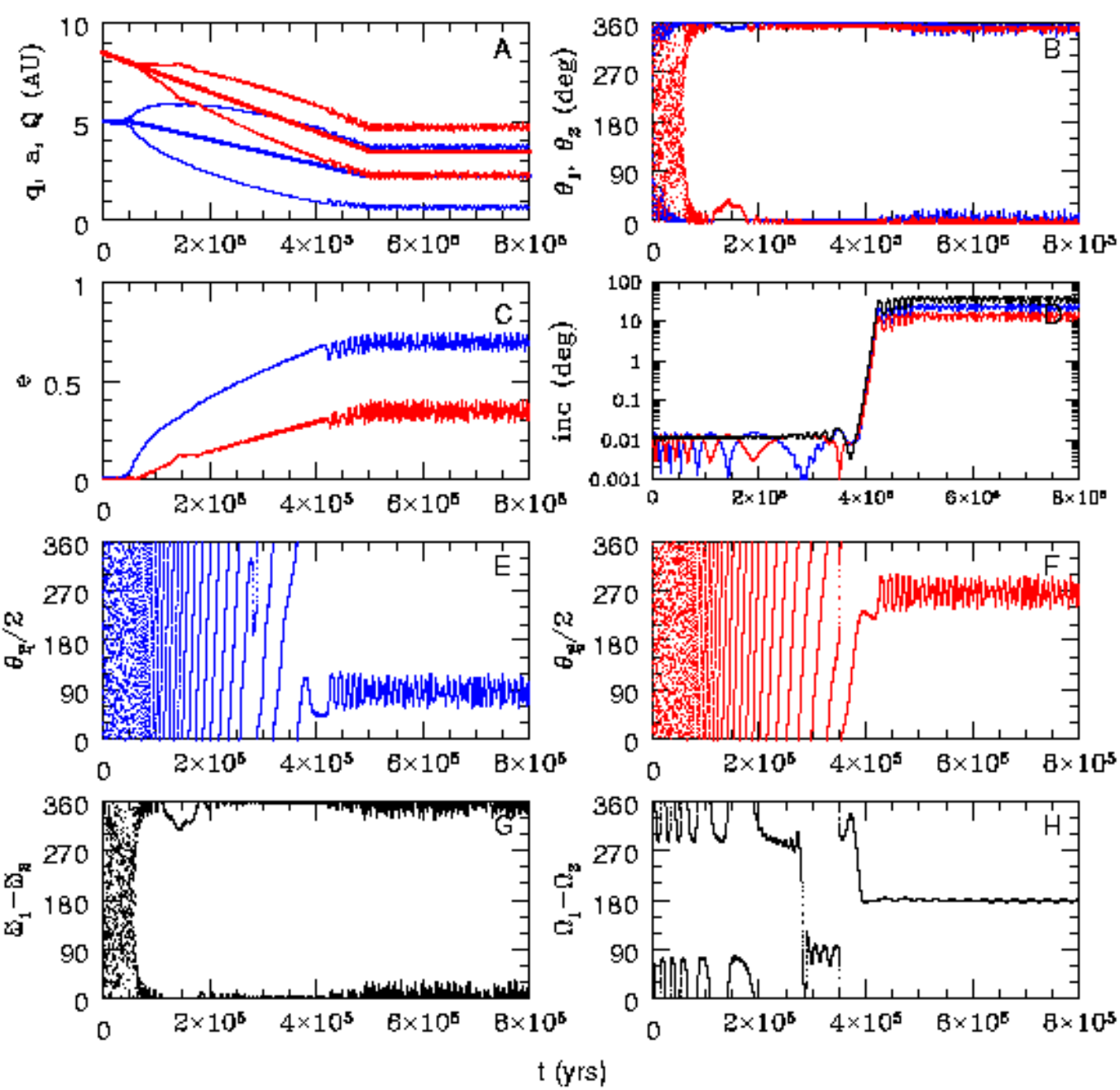

Fig. 10.- Another version of the baseline run (cf. Fig. 1), this time with migration turned off at $5 \times 10^{5}$ years. 
(2001). In this case, planets could keep migrating indefinitely without meeting a catastrophic end, though they would eventually move beyond the stellocentric radius accessible to radial velocity searches.

Finally, there is the intriguing possibility that inclination excitation could actually serve as a "parking mechanism" which puts an end to migration. One should keep in mind that we have used a simple, ad-hoc migration prescription in our simulations, nothing more than a constant, negative $\dot{a}$ which is enforced for the outer planet. The interaction of a significantly eccentric and inclined giant planet with a disk is in fact highly uncertain. One may speculate that a highly-inclined planet might be coupled less strongly to the surrounding disk. At the very least, by the time the planet's inclination exceeds that of the disk $\left(\sim 4^{\circ}\right.$ from midplane to scale height at $5 \mathrm{AU}$, decreasing inward for a flared disk), it may no longer be able to maintain a clean gap or edge. Such questions must ultimately be addressed through detailed hydrodynamic simulations, which as yet are restricted to the case of planets on circular, disk-coplanar orbits.

We thank Man Hoi Lee, Stan Peale, Geoff Marcy, Doug Lin and Steven Lubow for helpful and informative discussions. We also thank an anonymous referee for suggestions which helped us to improve the paper. We acknowledge support by UC Berkeley's Center for Integrative Planetary Science (EWT) and NASA's Origins of Solar Systems program through Grants NAG5-8861 (EWT) and 188-07-21-03-00 (JJL).

\section{REFERENCES}

Benedict, G. F., McArthur, B. E., Forveille, T., Delfosse, X., Nelan, E., Butler, R. P., Spiesman, W., Marcy, G., Goldman, B., Perrier, C., Jefferys, W. H., \& Mayor, M. 2002, ApJ, 581, L115

Bodenheimer, P., Hubickyj, O., \& Lissauer, J. J. 2000, Icarus, 143, 2

Bryden, G., Różyczka, M., Lin, D. N. C., \& Bodenheimer, P. 2000, ApJ, 540, 1091

Cabot, W., Canuto, V. M., Hubickyj, O., \& Pollack, J. B. 1987, Icarus, 69, 387

Chiang, E. I., \& Goldreich, P. 1997, ApJ, 490, 368

Cumming, A., Marcy, G. W., Butler, R. P., \& Vogt, S. S. 2003, in to appear in Scientific Frontiers in Research on Extrasolar Planets (ASP Conference Series)

Dubrulle, B. 1993, Icarus, 106, 59 
Duncan, M. J., Levison, H. F., \& Lee, M. H. 1998, AJ, 116, 2067

Gammie, C. F. 1996, ApJ, 457, 355

Goldreich, P. 1965, MNRAS, 130, 159

Goldreich, P., \& Sari, R. 2003, ApJ, submitted

Gomes, R. S. 2000, AJ, 120, 2695

Haisch, K. E., Lada, E. A., \& Lada, C. J. 2001, ApJ, 553, L153

Hayashi, C. 1981, Prog. Theor. Phys., 70, 35

Kalas, P., \& Jewitt, D. 1995, AJ, 110, 794

Kley, W. 2000, MNRAS, 313, L47

Laughlin, G., \& Chambers, J. E. 2001, ApJ, 551, L109

Lee, M. H., \& Peale, S. J. 2002, ApJ, 567, 596

—. 2003, AAS/Division on Dynamical Astronomy Meeting \# 34

Levison, H. F., Lissauer, J. J., \& Duncan, M. J. 1998, AJ, 116, 1998

Lin, D. N. C., Papaloizou, J. C. B., Terquem, C., Bryden, G., \& Ida, S. 2000, Protostars and Planets IV, 1111

Lissauer, J. J. 1987, in Planetary Geology and Geophysics Program Report, 100--

Lissauer, J. J., Peale, S. J., \& Cuzzi, J. N. 1984, Icarus, 58, 159

Lubow, S. H., \& Ogilvie, G. I. 2001, ApJ, 560, 997

Malhotra, R. 1998, in 29th Annual Lunar and Planetary Science Conference, March 16-20, 1998, Houston, TX, Vol. 29, 1476

Malhotra, R., \& Dermott, S. F. 1990, Icarus, 85, 444

Marcy, G. W., Butler, R. P., Fischer, D., Vogt, S. S., Lissauer, J. J., \& Rivera, E. J. 2001, ApJ, 556, 296

Masset, F., \& Snellgrove, M. 2001, MNRAS, 320, L55

Mizuno, H., Nakazawa, K., \& Hayashi, C. 1978, Prog. Theor. Phys., 60, 699 
Mouillet, D., Larwood, J. D., Papaloizou, J. C. B., \& Lagrange, A. M. 1997, MNRAS, 292, 896

Murray, C. D., \& Dermott, S. F. 1999, Solar System Dynamics (Cambridge University Press)

Nelson, R. P., Papaloizou, J. C. B., Masset, F., \& Kley, W. 2000, MNRAS, 318, 18

Papaloizou, J. C. B., Nelson, R. P., \& Masset, F. 2001, A\&A, 366, 263

Peale, S. J. 1976, ARA\&A, 14, 215

Pollack, J. B., Hubickyj, O., Bodenheimer, P., Lissauer, J. J., Podolak, M., \& Greenzweig, Y. 1996, Icarus, 124, 62

Rivera, E. J., \& Lissauer, J. J. 2001, ApJ, 558, 392

Snellgrove, M. D., Papaloizou, J. C. B., \& Nelson, R. P. 2001, A\&A, 374, 1092

Strom, S. E., Edwards, S., \& Skrutskie, M. F. 1990, in Cool Stars, Stellar Systems, and the Sun; Proceedings of the 6th Cambridge Workshop, Seattle, WA, Sept. 18-21, 1989 (A91-44876 19-90). San Francisco, CA, Astronomical Society of the Pacific, Vol. 6, $275-288$

Thommes, E. W., Duncan, M. J., \& Levison, H. F. 2003, Icarus

Thommes, E. W., \& Lissauer, J. J. 2003, in to appear in Proceedings of STSci Astrophsyics of Life Symposium, May 6-9, 2002 (ASP Conference Series)

Tittemore, W. C., \& Wisdom, J. 1989, Icarus, 78, 63

Wahhaj, Z., Koerner, D. W., Ressler, M. E., Werner, M. W., Backman, D. E., \& Sargent, A. I. 2003, ApJ, 584, L27

Ward, W. R. 1997, Icarus, 126, 261

Wisdom, J., \& Holman, M. 1991, AJ, 102, 1528

Yu, Q., \& Tremaine, S. 2001, AJ, 121, 1736 